\title{
Conocimientos, percepciones de riesgo y estigma sobre tuberculosis en población carcelaria de Cali, Colombia, 2020
}

\author{
Knowledge, risk perceptions and stigma around tuberculosis in prison \\ population in Cali, Colombia, 2020 \\ Lina Catalina Strba Idarraga ${ }^{1}$, Johana Alarcón Soto ${ }^{1}$, Karen Ahumada ${ }^{1}$, Jorge \\ Enrique Figueroa ${ }^{1,2}$, Yessenia Niño Ramírez ${ }^{1,3}$, Robinson Pacheco López ${ }^{1,4}$ \\ ${ }^{1}$ Grupo interdisciplinario de Investigación de Epidemiología y Salud pública, \\ Universidad Libre, Cali, Colombia \\ ${ }^{2}$ Universidad Icesi, Departamento de Estudios Sociales y Estudios Políticos \\ ${ }^{3}$ Secretaría De Salud Pública Municipal, Cali, Colombia \\ ${ }^{4}$ Universidad Libre, Grupo de Investigación en Epidemiología y servicios GRIEPIS, \\ Cali, Colombia
}

\begin{abstract}
Resumen
La Población Privada de la Libertad (PPL) es considerada como una población priorizada para el desarrollo de intervenciones del programa de tuberculosis (TB) al tener mayor riesgo de adquirirla. Así, se deben realizar intervenciones cuya estrategia sea la promoción de la salud y prevención de la enfermedad en los centros carcelarios de manera tal que se fortalezcan los conocimientos sobre $\mathrm{TB}$, se genere una alta percepción del riesgo de contraerla y disminuya el estigma. El objetivo del estudio es identificar los conocimientos, percepciones de riesgo y estigma sobre tuberculosis en una población carcelaria de Cali Colombia durante el 2020. El estudio es observacionaldescriptivo-transversal, con 138 personas sin tuberculosis de un centro penitenciario de Cali. Se realizó una encuesta validada por Ahumada et al (2021), para la medición del conocimiento, percepción del riesgo, estigma y variables sociodemográficas. La población tenía $39 \pm 8,79$ años, secundaria incompleta $(50,72 \%)$ y no se identificaron con alguna etnia $(66,67 \%)$. La mayoría identificó la TB como enfermedad pulmonar $(76,1 \%)$ y escuchó de ella por primera vez en la cárcel (73,19\%). El 94,20\% indicó que la TB puede causar la muerte; $29,71 \%$ iría a sanidad si se encontrara un compañero con TB. Se encontró un nivel bajo de estigma $(39,86 \%)$. Finalmente, la investigación mostró conocimientos básicos sobre TB, siendo que el "voz a voz" un punto clave para la identificación de TB dentro del establecimiento; se reconoció la posibilidad de muerte a
\end{abstract}

Fecha de envío: 30/07/2021

Fecha de aceptación: 30/08/2021 
causa de TB, así como la posibilidad de evitarla. Se encontró un bajo nivel de estigma que puede estar asociado a los niveles de conocimiento encontrados sobre TB.

Palabras clave: Tuberculosis, conocimientos, percepción del riesgo, estigma, población privada de la libertad, instrumento validado.

\begin{abstract}
The Population Deprived of Liberty (PPL) is considered a priority population for the development of tuberculosis (TB) program interventions as they have a higher risk of acquiring it. Thus, interventions whose strategy is the promotion of health and prevention of the disease in prisons should be carried out in such a way that knowledge about TB is strengthened, a high perception of the risk of contracting it is generated and stigma is reduced. The objective of the study is to identify the knowledge, risk perceptions and stigma about tuberculosis in a prison population of Cali, Colombia, during 2020. The study is observational-descriptive-cross-sectional, with 138 people without tuberculosis from a prison in Cali. A survey validated by Ahumada et al (2021). Was carried out to measure knowledge, risk perception, stigma and sociodemographic variables. The population was $39 \pm 8.79$ years old, incomplete high school (50.72\%) and they did not identify with any ethnic group (66.67\%). Most identified TB as a lung disease $(76.1 \%)$ and heard about it for the first time in prison (73.19\%). $94.20 \%$ indicated that TB can cause death; $29.71 \%$ would go to health if a partner with TB was found. A low level of stigma was found $(39.86 \%)$. Finally, the research showed basic knowledge about TB, with "voice to voice" a key point for the identification of TB within the establishment; The possibility of death from TB was recognized, as well as the possibility of avoiding it. A low level of stigma was found that may be associated with the levels of knowledge found about TB.
\end{abstract}

Keywords: Tuberculosis, knowledge, risk perception, stigma, population deprived of liberty, validated instrument.

\section{Introducción}

La tuberculosis (TB) es una enfermedad infecciosa crónica progresiva causada principalmente por el complejo Mycobacterium tuberculosis. Se transmite por la inhalación de partículas en suspensión en el aire que contienen la micobacteria y puede desarrollarse en cualquier órgano o tejido; sin embargo, la forma más común es la pulmonar. La TB continúa siendo un serio problema de salud pública a nivel mundial. Es una enfermedad infectocontagiosa de fácil transmisión, siendo una de las 10 principales causas de muerte en todo el mundo y, hasta mediados del año 2020, la principal causa de muerte por un agente infeccioso por encima del VIH/SIDA. Según el Global TB Report de la Organización Mundial de la Salud (OMS), durante el 2019 se enfermaron de tuberculosis alrededor de 10 millones de personas y 1,3 millones murieron por esta causa (1-2). 
En Colombia, el Sistema de Vigilancia en Salud Pública (SIVIGILA) notificó en el 201915.034 casos de tuberculosis de todas las formas y una tasa de incidencia de 27 casos por cada 100 mil habitantes. En el Valle del Cauca se presentaron para este mismo año un total de 1.913 casos (3). En Santiago de Cali, según el informe de la Secretaría de Salud Pública Municipal, en el 2019 se contó con 1.332 registros, de los cuales 1.284 corresponden a la condición sensible y 48 fueron resistentes. En el país existen poblaciones vulnerables y de riesgo con mayor carga de tuberculosis, los cuales, para este mismo año, fueron encabezados por la población privada de la libertad (36.67\%), habitantes de calle $(29.86 \%)$, población indígena $(15.63 \%)$, trabajadores de la salud $(8.42 \%)$ y migrantes $(5.81 \%)$. Estas son cifras importantes porque estos grupos, además del riesgo, deben sobrellevar condiciones de pobreza que no permiten garantías para el mantenimiento de la calidad de vida. Si sumamos esto a la enfermedad, llegamos a la reducción de ingresos familiares actuales y futuros, la disminución del rendimiento para trabajar o estudiar, y la intensificación de otros costos como transportes, traslados a clínicas/hospitales, medicamentos, equipos hospitalarios, entre otros, que deben asumir las familias (4-7).

Para hacer frente a la enfermedad, la Organización Mundial de la Salud (OMS) y la Organización Panamericana de la Salud (OPS) han adoptado diferentes estrategias en la lucha contra la TB, como el plan estratégico "Colombia hacia el fin de la TB 2016-2025", donde uno de sus objetivos es reducir el $50 \%$ de la incidencia, el
$75 \%$ de la mortalidad y el $60 \%$ de los costos catastróficos causados a los afectados por TB y sus familias (8-11).

Un aspecto por resaltar es que múltiples autores han reportado que el éxito de cualquier estrategia está en ampliar el conocimiento y cambiar la percepción de riesgo sobre la TB. Las actitudes y el desconocimiento (que incluye también los vacíos en la información y los conceptos erróneos) llevan a conductas estigmatizantes y de rechazo. Estos determinantes son especialmente relevantes en poblaciones vulnerables como habitantes de calle y población privada de la libertad, puesto que una mejora en los conocimientos y en las acciones de autocuidado puede contribuir a mitigar la incidencia de la tuberculosis en los grupos de riesgo. Al abordar los diferentes grupos sociales de manera individual y comunitaria se puede generar un impacto que tienda a mejorar las prácticas de autocuidado de las personas con TB, un aumento en la adherencia al tratamiento y una disminución en las cifras respecto al estigma de la enfermedad (12-16).

Por lo anterior, los centros penitenciarios se presentan como un escenario complejo debido a la interacción de factores individuales, programáticos, de conocimientos, dentro de un contexto sociopolítico, económico, educativo, laboral, que determinan el proceso saludenfermedad (17). Hay que reconocer, entonces, que la prevención es influenciada por el desconocimiento de la enfermedad, pero este desconocimiento a su vez podría llevar a la creación de mitos, estigmas, temores y estereotipos que se transmiten a los pacientes y sus familias (16). La pre- 
sente investigación buscó los conocimientos, percepciones de riesgo y estigma sobre tuberculosis en una población privada de la libertad de un centro carcelario de Cali Colombia durante el 2020, con el fin de brindar elementos para el apoyo a la toma de decisiones e intervenciones en las estrategias de promoción y prevención de la enfermedad en centros carcelarios.

\section{Materiales y método}

Se realizó un estudio observacional, descriptivo de corte transversal, utilizando un instrumento de recolección de información validado en 2020 por Ahumada et al (18), orientado a medir 3 dimensiones específicas: conocimientos, percepción de riesgo y estigma sobre tuberculosis. Para la recolección de la información se utilizó un instrumento de 17 ítems que miden tres dimensiones especificas: conocimientos, percepción de riesgo y estigmas sobre tuberculosis en población privada de la libertad. El instrumento fue validado mediante un consenso de expertos, y reporta un índice de validez del contenido $0.95 \%$ y confiabilidad por el alfa de Cronbach de 0.59. Se considera aceptable por ser un instrumento multidimensional (18); además, presenta 4 ítems adicionales con preguntas sociodemográficas. Para la utilización de dicho documento se contó con la autorización de su autor.

En el mes de julio de 2020, se invitó a participar a 138 personas sin diagnóstico de tuberculosis que se encontraban privados de la libertad en el establecimiento penitenciario de Villahermosa Cali, a quienes se les solicitó su consentimiento para la participación voluntaria de la investigación.

Para el cálculo del tamaño de la muestra se utilizó información aportada por la Secretaría de Salud Pública Municipal de Cali (SSPM) y su programa de Microbacterias, considerando los 12 patios con los que cuenta el establecimiento penitenciario y que albergaban una población total aproximada de 5.964, Población Privada de la Libertad (PPL), para la fecha de inicio del estudio. Se realizó el cálculo bajo el supuesto que toda la población era homogénea; se seleccionó el patio con una población estimada de $459 \mathrm{PPL}$, dado que la frecuencia esperada era muy variable según la literatura científica. Se estimó una frecuencia del $15 \% \pm 5$ para obtener el máximo tamaño de muestra y un límite de confianza del $5 \%$. El patio seleccionado obedeció también a las recomendaciones de la SSPM.

El cálculo del tamaño se realizó en la plataforma online Open Epi, aplicando la siguiente fórmula: $n=[E D F F * N p(1-$ $p)] /[(d 2 / Z 21-\alpha / 2 *(N-1)+p *(1-p)])$,

Tamaño de la muestra para la frecuencia en una población

Tamaño de la población (para el factor de corrección de la población finita o $\mathrm{fcp})(\mathrm{N})$ :

frecuencia \% hipotética del factor del resultado en la población (p):

Límites de confianza como \% de 100(ab- $5 \%$ soluto $+/-\%)(d)$ :

Efecto de diseño (para encuestas en 1 grupo-EDFF):

Se obtuvo una muestra de 138 participantes. La propuesta fue aprobada por el 
Tamaño muestral (n) para Varios Niveles de Confianza

$\begin{array}{ll}\text { Intervalo de Confian- } & \text { Tamaño de la mues- } \\ \text { za }(\%) & \text { tra } \\ 95 \% & 138\end{array}$

Comité de ética y bioética de investigación de la Universidad Libre seccional Cali.

Para la selección aleatoria de los participantes y la aplicación de la encuesta, estas se aplicaron en un solo momento para evitar la contaminación de la información.

Tras la obtención del aval de los directivos del INPEC, tuvo desarrollo esta actividad, en la que se contó con la colaboración de una profesional perteneciente al grupo de Microbacterias de la SSPM de Cali, el cual se encargó del acercamiento a los participantes y del diligenciamiento del cuestionario. El tiempo que tomó cada participante en realizar la encuesta fue de aproximadamente 10 minutos para responder los 17 ítems del formato, además de las 4 preguntas de tipo sociodemográfico.

Esta investigación fue aprobada como una investigación sin riesgo por el Comité de Ética de la Universidad Libre y según la Resolución 8430 de 1993 del Ministerio de la Protección Social de Colombia, la Resolución 01 del 10 de agosto de 2015, el Comité de Ética y Bioética de Investigación de la Universidad Libre, el día 2 de abril 2020.

Con la información recolectada se creó una base de datos utilizando el programa Excel 2019. Todos los análisis se desarrollaron utilizando el software SPSS. Se hizo uso de la estadística descriptiva; así, las variables cualitativas se resumieron con frecuencias y proporciones; se emplearon medidas de tendencia central y dispersión, mediante el uso del promedio y desviación estándar y la mediana, en el caso de las variables cuantitativas. Para el análisis bivariado se empleó la prueba de Chicuadrado y se consideró un nivel de significancia estadística $\mathrm{p}<0,05$, y se compararon las diferencias para las respuestas del nivel de estigma y las variables sociodemográficas. Considerando que el instrumento contiene preguntas abiertas, las respuestas a estas fueron agrupadas según criterios de codificación a partir de categorías emergentes, lo cual permitió establecer frecuencias con sus respectivos porcentajes.

Para el análisis de la variable estigma, al ser una variable de categoría subjetiva, no se necesitó un diagnóstico referente o estándar para medir el estigma, sino que bastó con la aplicación del instrumento de medición, en donde el participante respondía SÍ o NO a cada una de las preguntas realizadas. Por tener un total de 6 preguntas, el tener 0 respuestas con SÍ es clasificado sin estigma; entre 1 y 2 para bajo estigma; entre 3 y 4 para estigma medio; y entre 5 y 6 para estigma alto.

\section{Resultados}

En esta investigación participaron 138 personas con una edad promedio de 30 años, con un predominio de adultos jóvenes $(62,32 \%)$. El nivel de escolaridad más frecuente fue secundaria incompleta $(50,72 \%)$, seguido de secundaria completa $(23,91 \%)$. Respecto a la variable etnia, el $33,33 \%$ se identificaron dentro del 
grupo clasificado como negro(a), afrocolombiano(a), mulato(a) o afrodescendiente (tabla 1); además, también se encontró que el $100 \%$ de los participantes estaban afiliados a la salud bajo el régimen subsidiado.

De esta manera, se encontró que la mayoría identificaron la TB como una enfermedad pulmonar $(76,1 \%, \mathrm{n}=105 / 138)$, tal como se observa en la tabla 2. Esta categorización también se realizó para reconocer los síntomas de TB que identificaban las personas encuestadas, a lo que se encontró que el 78,3\% manifestaban la tos, seguido de la flema y fiebre, indicando la presencia de alto nivel de conocimientos respecto de la enfermedad (tabla 2).

En la evaluación del conocimiento también se indagó sobre la primera vez que se había escuchado información sobre la tuberculosis. Se encontró que la mayoría obtuvo esta información dentro de la cárcel $(73,19 \%, \mathrm{n}=101)$, y fue recibida principalmente de sus compañeros $(66,67 \%$, $\mathrm{n}=92$ ), como se muestra en la tabla 3 . Tal comportamiento resalta la importancia que juega el compañero como fuente de información dentro de esta comunidad, lo que además podría considerarse como una alternativa para futuras intervenciones en pro del mejoramiento del conocimiento. También se encontró que el 95,65\% (n=132) creían que la TB sí se puede contagiar. Solo el 2,17\% $(\mathrm{n}=3)$ no sabían o respondieron que no se contagia $(2,17 \%, \mathrm{n}=3)$. A estas cifras se suma el que el $75,36 \%(\mathrm{n}=104)$ manifestó que la tuberculosis tiene cura, seguido de un $15,22 \%(\mathrm{n}=21)$ que creía que no y un $9,42 \%(n=13)$ que no sabía sobre el tema.

Finalmente, sobre el conocimiento se preguntó cómo creían que se daban los casos de contagio de tuberculosis dentro de la cárcel, a lo que la mayoría contestó que se producían por transmisión aérea $(46,4 \%)$, seguido de aquellos que manifestaron que se daba al compartir los elementos personales $(42,0 \%)$, y que la mejor forma de prevención era la automedicación $(42,2 \%)$. Un aspecto por resaltar es que el $17,7 \%$ refirió que los hábitos saludables eran una forma de prevención del contagio (tabla 4).

Con relación a la percepción del riesgo, prevalecieron las personas que indicaron que la tuberculosis sí puede causar la muerte $(94,20 \%)$ y las que contestaron que creen que la TB sí se puede evitar, como aparece en la tabla 5. A estas percepciones se debe adicionar que el $29,71 \%$ se dirigiría a sanidad en los casos de encontrarse con un compañero de patio con TB o que tomarían distanciamiento $(23,91 \%)$, lo cual es evidente en la tabla 6 . El estigma y las valoraciones obtenidas a través de 6 diferentes preguntas se encuentran discriminadas en la tabla 7, aunque, a groso modo, los niveles encontrados fueron bajos. Además, la encuesta aplicada permite sacar una escala que entrega el nivel de estigma entre los encuestados, lo que para este caso mostró, de manera general, niveles bajos de estigma $(39,86 \%, \mathrm{n}=55)$, aunque los niveles medios y altos se presentaron en proporciones bastante similares al primero (tabla 8 ).

En una siguiente etapa se hizo un análisis bivariado para encontrar relaciones entre la variable dependiente "nivel de estigma" y las variables sociodemográficas. Se encontraron solo diferencias estadísticas significativas $(\mathrm{p}=0,021)$ al relacionar 
Tabla 1: Variables sociodemográficas de la población de estudio

\begin{tabular}{llll}
\hline Características & Escala & N & \% \\
\hline \multirow{4}{*}{ Edad } & 18 a 30 años & 86 & 62,32 \\
& 31 a 40 años & 35 & 25,36 \\
& 41 a 50 años & 13 & 9,42 \\
& Mayores de 50 & 4 & 2,90 \\
& Ninguno & & \\
& Primaria completa & 1 & 0,72 \\
& Primaria incompleta & 10 & 14,49 \\
Nivel de escolaridad & Secundaria completa & 33 & 23,91 \\
& Secundaria incompleta & 70 & 50,72 \\
& Técnico & 1 & 0,72 \\
& Tecnológico & 3 & 2,17 \\
& & & \\
Etnia & Negro, mulato, afro & 46 & 33,33 \\
& Otro & 92 & 66,67 \\
\hline
\end{tabular}

la etnia y el nivel de estigma. Se mostró que los niveles altos de estigma se presentan en el grupo de personas identificadas como negro, mulato, afrodescendientes o afrocolombianos $(30,43 \%)$. En la siguiente tabla se encuentran los distintos cruces realizados.

Para la percepción de riesgo que fue evaluada con dos preguntas también se buscó la existencia de relación con la edad, el nivel educativo y la etnia (tablas $10 \mathrm{y}$ 11). En ninguno de los casos se encontraron diferencias estadísticas significativas (p¿0,005). Aun así, tanto para la edad como para el nivel educativo y la etnia, los grupos que los conformaban tenían una distribución similar. Es decir, con respecto a la edad, tanto los menores de 28 años como los mayores a esta edad, y en la etnia, los negros, mulatos, afro, etc. y los clasificados como otros, presentaron una proporción similar al creer que la tuberculosis puede causar la muerte y que se puede evitar. Con relación al nivel educativo, las proporción de personas con solo primaria y superior a ella es similar respecto a la creencia de que la tuberculosis puede causar la muerte, aunque sí difiere un poco en la creencia que la tuberculosis se puede evitar en la cárcel (hasta primaria, $48,39 \%$, y superior a primaria, $66,36 \%$ ).

\section{Discusión}

Este es el primer estudio en identificar los conocimientos, la percepción de riesgo y el estigma en personas privadas de la libertad de un centro carcelario de Cali en el año 2020, a través de una encuesta validada. Es prioritario establecer una línea de base sobre estos temas, puesto que los centros penitenciarios son espacios de al- 
Tabla 2: Respuestas de las preguntas abiertas relacionadas con el conocimiento general sobre TB

\begin{tabular}{llll}
\hline Pregunta & Categorías & N & \% \\
\hline & Enfermedad pulmonar & 105 & 76,1 \\
& Muerte & 10 & 7,2 \\
& No sabe & 8 & 5,8 \\
& Otras & 7 & 5,1 \\
& Enfermedad infectocontagiosa & 6 & 4,3 \\
& Hemoptisis & 5 & 3,6 \\
& Sistema inmune & 4 & 2,9 \\
& Gripa & 4 & 2,9 \\
& Pérdida de peso & 3 & 2,2 \\
& Pérdida del apetito & 2 & 1,4 \\
& & & \\
& Tos & 108 & 78,3 \\
& Flema & 47 & 34,1 \\
& Fiebre & 36 & 26,1 \\
& Pérdida de peso & 27 & 19,6 \\
& Fatiga o adinamia & 27 & 19,6 \\
& Sudoración & 24 & 17,4 \\
& Hemoptisis & 18 & 13,0 \\
& Dificultad respiratoria & 13 & 9,4 \\
& Pérdida de apetito & 11 & 8,0 \\
& No sabe & 9 & 6,5 \\
& Alteración de sueño & 7 & 5,1 \\
& Cefalea & 4 & 2,9 \\
& Escalofrío & 4 & 2,9 \\
& Otras & 4 & 2,9 \\
\hline
\end{tabular}

Tabla 3: Información sobre TB

\begin{tabular}{llll}
\hline Pregunta & Categorías & N & \% \\
\hline \multirow{2}{*}{ ¿Dónde escuchó por primera vez informa- } & En la cárcel & 101 & 73,19 \\
ción sobre la TB? & Fuera de la cárcel & 34 & 24,64 \\
& No ha escuchado & 3 & 2,17 \\
& & & \\
& Compañeros & 92 & 66,67 \\
En la cárcel, ¿de quién recibió información & Personal sanitario & 48 & 34,78 \\
sobre TB? & Promotor & 24 & 17,39 \\
& Carteleras & 23 & 16,67 \\
& Guardianes & 1 & 0,72 \\
\hline
\end{tabular}


Tabla 4: Preguntas abiertas relacionadas con el conocimiento

\begin{tabular}{|c|c|c|c|}
\hline Pregunta & Categorías & $\mathbf{n}$ & $\%$ \\
\hline \multirow{8}{*}{$\begin{array}{l}\text { Cómo creen los en- } \\
\text { cuestados que se dan } \\
\text { los casos de contagio } \\
\text { de tuberculosis den- } \\
\text { tro de la cárcel }\end{array}$} & Transmisión por vía aérea & 64 & 46,4 \\
\hline & Compartiendo los elementos personales & 58 & 42,0 \\
\hline & Compartiendo tabaco y/o sustancias psicoactivas & 39 & 28,3 \\
\hline & No sabe & 2 & 1,4 \\
\hline & Sistema inmunológico bajo & 2 & 1,4 \\
\hline & Sangre & 1 & 0,7 \\
\hline & No usar tapabocas & 1 & 0,7 \\
\hline & Relaciones sexuales & 1 & 0,7 \\
\hline \multirow{11}{*}{$\begin{array}{l}\text { Cómo creen los en- } \\
\text { cuestados que se pre- } \\
\text { viene el contagio de } \\
\text { tuberculosis }\end{array}$} & Automedicación & 60 & 42,2 \\
\hline & Nada & 30 & 21,1 \\
\hline & Hábitos Saludables & 25 & 17,7 \\
\hline & No Sabe & 10 & 6,8 \\
\hline & Medidas De Prevención & 5 & 3,4 \\
\hline & No Fumar & 4 & 2,7 \\
\hline & No Compartir Elementos Personales & 3 & 2,0 \\
\hline & Religión & 3 & 2,0 \\
\hline & Uso De Tapabocas & 1 & 0,7 \\
\hline & Distanciamiento Social & 1 & 0,7 \\
\hline & Higiene Personal & 1 & 0,7 \\
\hline
\end{tabular}

Tabla 5: Percepción del riesgo de TB

\begin{tabular}{llll}
\hline Variable & Escala & N & \multicolumn{1}{c}{$\%$} \\
\hline \multirow{2}{*}{ Cree que la TB puede causar la muerte } & Sí & 130 & 94,20 \\
& No & 2 & 1,45 \\
& No sé & 6 & 4,35 \\
Cree que la TB se puede evitar & & & \\
& Sí & 86 & 62,32 \\
& No & 47 & 34,06 \\
& No sé & 5 & 3,62 \\
\hline
\end{tabular}

to riesgo de infección para $\mathrm{TB}$ debido a que conjugan condiciones propicias para el contagio, como la frecuencia de exposición, su duración, las difíciles condiciones ambientales y la limitada aplicación de prácticas saludables. Los datos releva- 
Tabla 6: Preguntas abiertas relacionadas con la percepción del riesgo

\begin{tabular}{llll}
\hline Pregunta & Categorías & $\mathbf{n}$ & $\mathbf{\%}$ \\
\hline & Dirigirse a sanidad & 41 & 29,7 \\
& Toman distanciamiento & 33 & 23,9 \\
& Dirigirse a los Promotores Salud & 24 & 17,4 \\
Qué haría si un compañero de & Dirigirse al líder de patio & 13 & 9,4 \\
patio tiene TB & Brindar apoyo emocional & 13 & 9,4 \\
& No compartir elementos personales & 10 & 7,2 \\
& Automedicación & 9 & 6,5 \\
& Nada & 3 & 2,2 \\
& No compartir cigarrillo & 1 & 0,7 \\
\hline
\end{tabular}

Tabla 7: Valoraciones relacionadas con el estigma

\begin{tabular}{|c|c|c|c|}
\hline Variable & Escala & $\mathrm{N}$ & $\%$ \\
\hline Las personas enfermas con TB tienen más limitacio- & Sí & 48 & 34,78 \\
\hline \multirow{2}{*}{ nes para desempeñar sus actividades diarias } & No & 90 & 65,22 \\
\hline & Sí & 56 & 40,58 \\
\hline \multirow[t]{2}{*}{$\begin{array}{l}\text { Usted le tendría miedo a alguien que está enfermo } \\
\text { de TB }\end{array}$} & No & 82 & 59,42 \\
\hline & Sí & 96 & 69,57 \\
\hline \multirow[t]{2}{*}{$\begin{array}{l}\text { Usted cree que las personas se sienten incómodas al } \\
\text { estar cerca de alguien enfermo de TB }\end{array}$} & No & 42 & 30,43 \\
\hline & Sí & 41 & 29,71 \\
\hline \multirow[t]{2}{*}{$\begin{array}{l}\text { Usted cree que se debe sentir lástima por las perso- } \\
\text { nas enfermas de TB }\end{array}$} & No & 97 & 70,29 \\
\hline & Sí & 85 & 61,59 \\
\hline \multirow[t]{2}{*}{$\begin{array}{l}\text { Usted cree que las personas con TB en la cárcel sien- } \\
\text { ten temor de contarle a los demás que padecen esta } \\
\text { enfermedad }\end{array}$} & No & 53 & 38,41 \\
\hline & Sí & 110 & 79,71 \\
\hline $\begin{array}{l}\text { Usted cree que las personas con TB dentro de la } \\
\text { cárcel deberían estar en un patio solo para ellos }\end{array}$ & No & 28 & 20,29 \\
\hline
\end{tabular}

dos en esta investigación pueden aplicarse para la generación de estrategias centra- das en el usuario que limiten el riesgo de infección en espacios de alto hacinamiento 
Tabla 8: Niveles de estigma encontrados entre las personas incluidas en la investigación

\begin{tabular}{llll}
\hline Variable & Escala & $\mathrm{N}$ & $\%$ \\
\hline \multirow{3}{*}{ Nivel de estigma } & Sin estigma & 3 & 2,20 \\
& Bajo estigma & 54 & 39,10 \\
& Estigma medio & 45 & 32,60 \\
& Estigma alto & 36 & 26,10 \\
\hline
\end{tabular}

Tabla 9: Relaciones entre la variable nivel de estigma y las variables sociodemográficas

\begin{tabular}{llllll}
\hline \multirow{2}{*}{ Variable } & \multicolumn{2}{l}{ Nivel de estigma, n (\%) } & & & \\
& Sin estigma & Estigma bajo & Estigma medio & Estigma alto & $\mathbf{p}^{*}$ \\
\hline Edad & & & & & \\
Menor o igual a 28 años & $2(3,33)$ & $19(31,67)$ & $20(33,33)$ & $19(31,67)$ & 0,3271 \\
$\begin{array}{l}\text { Mayores de 28 años } \\
\text { Nivel educativo }\end{array}$ & $1(1,28)$ & $35(44,87)$ & $25(32,05)$ & $17(21,79)$ & \\
$\begin{array}{l}\text { Primaria } \\
\text { Superior y técnica }\end{array}$ & $1(3,23)$ & $9(29,03)$ & $10(32,26)$ & $11(35,48)$ & 0,4574 \\
Etnia & $2(1,87)$ & $45(42,06)$ & $35(32,71)$ & $25(23,36)$ & \\
Negro, mulato, afro & $0(0,0)$ & $24(52,17)$ & $8(17,39)$ & $14(30,43)$ & 0,021 \\
Otro & $3(3,26)$ & $31(33,70)$ & $37(40,22)$ & $21(22,83)$ & \\
\hline
\end{tabular}

Tabla 10: Relaciones entre la variable percepción de riesgo y las variables sociodemográficas

\begin{tabular}{|c|c|c|c|c|}
\hline \multirow{2}{*}{ Variable } & \multicolumn{3}{|c|}{$\begin{array}{l}\text { Usted cree que la tuberculosis puede } \\
\text { causar la muerte, n }(\%)\end{array}$} & \multirow{2}{*}{$\mathrm{p}^{*}$} \\
\hline & Sí & No & No sé & \\
\hline \multicolumn{5}{|l|}{ Edad } \\
\hline Menor o igual a 28 años & $55(91,67)$ & $2(3,33)$ & $3(5,00)$ & \multirow{2}{*}{0,2495} \\
\hline Mayores de 28 años & $75(96,15)$ & $0(0,00)$ & $3(3,85)$ & \\
\hline \multicolumn{5}{|l|}{ Nivel educativo } \\
\hline Primaria & $29(93,55)$ & $1(3,23)$ & $1(3,23)$ & \multirow{2}{*}{0,6101} \\
\hline Superior y técnica & $101(94,39)$ & $1(0,93)$ & $5(4,67)$ & \\
\hline \multicolumn{5}{|l|}{ Etnia } \\
\hline Negro, mulato, afro & $45(97,83)$ & $0(00,00)$ & $1(2,17)$ & \multirow{2}{*}{0,3973} \\
\hline Otro & $85(92,39)$ & $2(2,17)$ & $5(5,43)$ & \\
\hline
\end{tabular}

$(19-20)$.

Bajo este panorama, se torna imperativo fortalecer algunos conocimientos básicos sobre la TB. El conocimiento sobre es- ta enfermedad es limitado en la mayoría de la población. El estudio de Adane et al (14) mostró que solo el $24 \%$ de su población contaba con conocimientos bási- 
Tabla 11: Relaciones entre la variable percepción de riesgo y las variables sociodemográficas

\begin{tabular}{lllll}
\hline & \multicolumn{2}{l}{$\begin{array}{l}\text { Usted cree que en la cárcel la } \\
\text { tuberculosis se pueda evitar, } \mathbf{n}\end{array}$} & \\
Variable & $\begin{array}{l}\text { ( \%) } \\
\text { Sí }\end{array}$ & No & No sé & p* \\
\hline Edad & & & & \\
$\begin{array}{l}\text { Menor o igual a 28 años } \\
\text { Mayores de 28 años }\end{array}$ & $37(61,67)$ & $20(33,33)$ & $3(5,00)$ & 0,7486 \\
Nivel educativo & $49(62,82)$ & $27(34,62)$ & $2(2,56)$ & \\
$\begin{array}{l}\text { Primaria } \\
\text { Superior y técnica }\end{array}$ & $15(48,39)$ & $15(48,39)$ & $1(3,23)$ & \multirow{2}{*}{0,1594} \\
Etnia & $71(66,36)$ & $32(29,91)$ & $4(3,74)$ & \\
Negro, mulato, afro & $30(65,22)$ & $15(32,61)$ & $1(2,17)$ & 0,7651 \\
Otro & $56(60,87)$ & $32(34,78)$ & $4(4,35)$ & \\
\hline
\end{tabular}

cos de la enfermedad, mientras que Figueroa et al (21) indicaron que más del $80 \%$ reconoció conocer poco o nada sobre la TB. Lo más común es encontrarla mal asociada con otras enfermedades desencadenantes (20) y a la exposición al viento frío (14). Pese a ello, los participantes de estas investigaciones resaltan diversos aspectos sobre el tema. Se puede destacar su capacidad de referirla como una enfermedad peligrosa, especialmente cuando aún no ha sido diagnosticada, que muestra signos y síntomas claros de la enfermedad como la tos y la fiebre, y por tanto requiere de cuidado (22), o como sucedió en la investigación de Cramm et al, en la que el $98 \%$ contestó de manera correcta que es una enfermedad que hoy se puede curar fácilmente con el tratamiento adecuado (20). Para el caso del estudio de Arroyo et al, el principal vacío de conocimiento estuvo relacionado con el modo de transmisión: los medios más referidos fueron contacto con personas que tienen la enfermedad $(27,9 \%)$, vía oral $(19,8 \%)$ y tos $(32,6 \%)$, y resultaron con porcentajes bajos. Adicionalmente, los participantes señalaron erróneamente como medios de trasmisión las toallas-peines $(17,4 \%)$ y a los mosquitos (1,2\%) (23).

En el presente estudio, se encontró que la PPL reconoce que la TB es una enfermedad de tipo pulmonar $(76,1 \%)$ y que la tos es uno de los principales síntomas que la acompañan (78,3\%). Además, manifiestan que la automedicación (42,2\%) es la mejor forma de prevenir su contagio, aunque evidentemente no es una práctica recomendada. Pese a que la automedicación no es una forma de prevención, sino una respuesta a la enfermedad, estos hallazgos posicionan a la PPL del centro carcelario de Villahermosa de la ciudad de Cali como un grupo con buenos niveles de conocimiento. Sin embargo, llama la atención que esta información se esté obteniendo principalmente por parte de los propios compañeros de patio $(66,67 \%)$, en 
vez de ser recibida por el personal de salud $(34,78 \%)$. Además, esta población obtuvo información por primera vez sobre la TB dentro de la cárcel $(73,19 \%)$. Por su parte, otras investigaciones reportaban la televisión, la radio y el personal de la salud como vías de obtención de la información sobre TB, las cuales se asociaron con mejores conocimientos y percepción de la TB (23), lo cual indica la posibilidad de aprovechar estos medios dentro del centro penitenciario.

Estos hallazgos indican que en la cárcel hay una circulación significativa de información sobre esta enfermedad entre la población carcelaria, lo cual evidencia la importancia de fortalecer la inducción y capacitación que reciben sobre la TB para que el "voz a voz" entre los compañeros de patio se fortalezca con los conocimientos y recomendaciones adecuadas. Es decir, aprovechar los canales de comunicación que existen entre la PPL para transmitir aquellos saberes necesarios para reducir los contagios y evitar aquellas prácticas que conducen a hábitos poco saludables y aquellas que generan discriminación.

Respecto a los conocimientos sobre síntomas y formas de contagio dentro del centro penitenciario, se presentan valores similares a lo encontrado en investigaciones como la de Figueroa et al, en donde las personas identificaron la tos $(75 \%)$ y las vías respiratorias $(61 \%)$ como factores de riesgo para la transmisión de la TB (21). No obstante, la riqueza y aporte que tiene la información obtenida en este estudio radica en que, a diferencia de los hallazgos de Figueroa et al, quienes emplearon preguntas cerradas que limitaban las opciones de respuesta, en esta inves- tigación se valoraron algunos conocimientos a través de preguntas abiertas, lo que reveló una diversidad importante de respuestas sin la posibilidad de opciones previas, con el fin de explorar las asociaciones que hace la PPL con la TB a partir de su imaginario social, percepciones propias y cosmovisión.

Una alta percepción del riesgo de contraer TB puede influir positivamente en la decisión de buscar atención médica y en la adherencia al tratamiento antituberculoso. Según el estudio de Cramm et al, el $95 \%$ de los entrevistados creía que las personas con tuberculosis ocultaban su enfermedad por miedo a lo que otros puedan decir, lo que al final se convierte en un problema para la adherencia y detección de casos (20). En este estudio, la percepción del riesgo que se obtuvo fue favorable, ya que el 94,20\% indicó que la TB puede causar la muerte, lo que lleva a que el $29,71 \%$ acudan a la enfermería al estar cerca de un compañero con TB, con lo cual se demuestra la existencia de un nivel de conciencia sobre la forma de manejar la enfermedad.

En la investigación de Adane et al, que también fue realizada entre población carcelaria, la tendencia fue encontrar actitudes favorables $(41 \%)$ y buenas prácticas $(55 \%)$, las cuales se veían beneficiadas por variables como la procedencia del sujeto, pues los residentes urbanos solían tener mejores conocimientos (OR ajustado $=2,16$; IC del $95 \%=1,15-4,06)$; y el nivel de escolaridad, puesto que los analfabetos llegaron a tener menos conocimientos (OR ajustado $=0,17$; IC del $95 \%=0,06-0,46)$, menos probabilidades de tener una actitud favorable (OR ajustado $=0,31 ; \mathrm{IC}$ del 
$95 \%=0,15-0,64)$ y menos buenas prácticas (ajustado OR $=0,35$; IC del $95 \%=$ 0,18-0,69) (14).

En esta investigación la percepción del riesgo no se encontró asociada con variables como la escolaridad, la edad y la etnia ( $p>0,05$ ); no obstante, en la asociación entre el nivel de estigma y el reconocimiento de la etnia sí se encontraron diferencias estadísticas significativas que indican que quienes se identificaron con la etnia negro(a), mulato(a), afrocolombiano(a) o afrodescendiente presentaron niveles más altos de estigma hacia la TB $(p=0,021)$. Una manera para explicar esta asociación radica en comprender las condiciones en las que vive la población afrodescendiente en nuestro país y en una ciudad como Cali, donde se enfrentan a un racismo estructural y una desigualdad histórica que los ha colocado en condiciones de vulnerabilidad como el acceso limitado a una educación de calidad y a empleos dignos. Arroyo et al indica que existe una discriminación racial que afecta de manera directa a la población afrocolombiana de Cali, ciudad que se caracteriza por tener el mayor número de población negra en Colombia (542.000 personas, que representan el $26 \%$ de los habitantes de la ciudad), según el censo de población de 2005 del DANE. Los resultados de dicha investigación dejaron ver que existe un 13,32\% de discriminación hacia la población afrocolombiana de Cali, la cual no se asocia a características objetivas del individuo (24). A lo anterior se suma que Bruyneel et al expone las duras condiciones de la población habitante del Pacífico colombiano, quienes son predominantemente afrocolombianos, y que, entre muchos otros factores, debido al abandono del Estado, se han visto en la necesidad de migrar hacia centros urbanos como la ciudad de Cali, llegando a sitios donde su condición de vulnerabilidad se intensifica y difícilmente pueden sustentarse a ellos o a sus familias de manera satisfactoria (25). En ese sentido, las condiciones estructurales colocan a la población afrodescendiente en un lugar de desventaja social que los hace menos acreedores de saberes y conocimientos para prevenir la generación de conductas estigmatizadoras hacia las personas con TB.

Resultados como los de Cramm et al mostraban que el $95 \%$ de la población encuestada sin tuberculosis creía que las personas con TB ocultaban su estado de TB porque tenían miedo de lo que otros pudieran decir. El $90 \%$ creía que "los pacientes que no toman su tratamiento son los culpables de propagar la TB", el $74 \%$ creía que las personas que contraían tuberculosis por beber o fumar, y el $51 \%$ refirió que si tenían tuberculosis la gente no los respetaría (20). Lo anterior es totalmente diciente si se considera que en esta investigación cerca de 1 de cada 3 PPL cree que la TB lleva a limitaciones en las actividades diarias, miedo de parte de sus compañeros, incomodidad al estar cerca de un paciente con TB, temor a decir que están enfermos y hasta la necesidad de patios diferentes para las personas contagiadas. Aquí se puede resaltar que Muegues et al manifestó que el control de la TB (de tipo pulmonar) en Villahermosa debe abordarse desde la perspectiva de los determinantes de la salud, mejorando las condiciones ambientales y nutricionales de los PPL y su acceso a los servicios de salud, lo que evidentemente llevaría a 
mejorar los niveles de estigma encontrados en este estudio (estigma alto en el $25,36 \%$ ), aunque no se debe olvidar que existen dentro de las cárceles una alta tasa de rotación de la PPL, que puede limitar los niveles de éxito en el reclutamiento, diagnóstico, tratamiento y seguimiento de estos pacientes (26) y lo que lleva a que se requiera un mayor nivel de capacitación constante.

A pesar de los esfuerzos que realizan los profesionales de la salud, en las prisiones el concepto de salud es a menudo olvidado o considerado de baja prioridad y, aunque el sistema de salud considera esta población dentro de sus planes de promoción y prevención, existen barreras que no permiten su cobertura a satisfacción, entre ellas el clima de desorganización y violencia que obstaculiza el acceso a la atención médica (27).

\section{Limitaciones $\mathrm{y}$ fortale- zas}

Entre las fortalezas se contó con un instrumento validado para medir conocimientos, percepciones y estigma al momento de elaborar las encuestas. Asimismo, el tipo de diseño que se escogió permitió responder la pregunta planteada al iniciar este proyecto. El tipo de estudio permitió obtener resultados en tiempos razonables y a corto plazo, además ser un estudio de bajo costo en comparación con otro tipo diseños de estudio. El estudio permite también explorar posibles asociaciones que posteriormente pueden estudiarse con mayor rigor a través de un diseño de estudio diferente. Con los resultados que arrojó la investigación se pueden planear y evaluar intervenciones de salud pública. Finalmente, este es el primer estudio que se hace utilizando un instrumento de medición de conocimiento, percepción de riesgo y estigma, siendo un punto de partida para aplicarlo en diferentes escenarios.

Entre las limitaciones se halla el haber atravesado la pandemia por COVID-19 al momento de aplicar las encuestas en el centro carcelario. A su vez, otra limitación es respecto al grupo de personas que generalmente siente temor a brindar respuestas a causa de las difíciles condiciones de la prisión o por la intimidación de otros compañeros, lo que puedo generar sesgos. La encuesta no contaba con un número suficiente de preguntas de tipo sociodemográfico que permitiera buscar asociaciones que dieran una mayor profundidad a los resultados y brindar la posibilidad de realizar más comparaciones con otros estudios.

\section{Conclusiones}

En relación con el conocimiento general sobre la tuberculosis, se encontró que la mayoría de las PPL $(76.1 \%)$ la reconoce como una patología pulmonar. Cabe resaltar el trabajo generado por el programa de tuberculosis la SSPM, por lo que se obtuvieron resultados generales de la enfermedad, pero no específicos, adecuados sobre la TB. Esto se podría complementar con la mejora de las condiciones de vida, en cuanto a su estructura, mejorando el hacinamiento, higiene, ventilación, entre otros.

La percepción del riesgo obtuvo buenos 
resultados, pues los PPL reconocen la posibilidad de muerte a causa de la TB pero, al tiempo, la posibilidad de evitarla, especialmente mediante ayuda profesional especializada, como a través de los encargados del área de sanidad y promotores de salud.

Al abordar el tema del estigma, aún prevalecen algunos aspectos asociados a este, como el miedo y la incomodidad a estar cerca a alguien enfermo, las limitaciones de las personas enfermas con TB para desempeñar actividades diarias y la posibilidad de ofrecer un patio diferente para este grupo.

Es de resaltar que, de manera general, los niveles de estigma son bajos, lo que podría estar asociado a los buenos niveles de conocimiento encontrados sobre la enfermedad y que parecen haber perpetuado dentro de la institución. Esta variable fue apropiadamente identificada con el instrumento aquí aplicado, lo cual deja ver la utilidad de su aplicabilidad en un grupo de tan alto riesgo como las PPL.

El instrumento aplicado, además de dar respuesta a la pregunta de investigación, nos permitió conocer mas a fondo la población a estudio, personas que han sido apartadas de su entorno habitual y que han creado dentro del centro penitenciario su propia cultura, sus "leyes", su lenguaje o jerga, y como al cambiar su condición de vida estas generan impacto sobre su salud física $\mathrm{y} / \mathrm{o}$ mental.

\section{Recomendaciones}

Entre las recomendaciones tenemos el que deba continuarse fortaleciendo el pro- grama de micobacterias de la SSPM, desde una política educativa a fin de fomentar el autocuidado y el reporte oportuno de los signos y síntomas de TB ante el área de sanidad, en busca de una captación y diagnostico oportuno, incluyendo tanto a la población TB como aquella que no presenta la enfermedad, además de vincular otros programas como VIH-SIDA, programas de salud mental y de sustancias psicoactivas y alcoholismo.

Asimismo, recomendamos promover la capacitación continua a los "líderes" que se encuentren dentro de la cárcel sobre diferentes temas de interés en salud pública, para ser estos replicadores de la información y así fortalecer los conocimientos de la población carcelaria. Con esto se lograría mejorar medidas de protección, poder cortar la cadena de transmisión de cualquier enfermedad, para sus compañeros, personal de guardia y sus familias, mejorar las percepciones de riesgo y los niveles de estigma.

Recomendamos tomar como referencia la presente investigación como base para realizar otros estudios en diferentes centros penitenciarios del país en busca de herramientas que permitan mejorar el nivel de conocimientos, la percepción y el estigma sobre la tuberculosis, además de que serviría para realizar comparaciones entre los hallazgos.

Finalmente, teniendo en consideración la utilidad del formato de recolección aquí utilizado, creemos que es una oportunidad para empezar a implementarlo considerando diferentes escenarios o grupos poblacionales. 


\section{Referencias}

(1) OMS - Organización Mundial de la Salud. ¿Qué es la tuberculosis y cómo se trata? 2016 [Consultado el 27 Nov 2020]. Disponible en: https: //www.who.int/features/qa/08/es/

(2) Adhamon T. Global tuberculosis report 2020. 2020 [Consultado el 21 En 2021]. Recuperado de: apps.who. int/iris/bitstream/handle/10665/ 336069/9789240013131-eng.pdf

(3) Colombia - Ministerio de Salud. Boletín Epidemiológico Semanal, semana 52. [Consultado el 5 Abr 2021]. Disponible en: https://www.ins.gov.co/buscadoreventos/BoletinEpidemiologico/ 2019_Boletin_epidemiologico_ semana_52.pdf

(4) Colombia - Ministerio de Salud. Tuberculosis Colombia 2019, Informe del evento. 2019. [Consultado el 22 Dic 2020]. Disponible en: https://www.ins.gov.co/buscadoreventos/Informesdeevento/ TUBERCULOSIS_2019.pdf

(5) Colombia - Ministerio de Salud. ¿Qué es tuberculosis (TB)? [Internet]. 2018. [Consultado el $15 \mathrm{Nov}$ 2020]. Disponible en: https://www. minsalud.gov.co/salud/publica/ PET/Paginas/Tuberculosis.aspx

(6) Sustain M. informe de vigilancia en salud pública de tuberculosis, hasta el periodo epidemiológico 13, Santiago de Cali, 2019. [Consultado el 21 Nov 2020].
Disponible en: http://scioteca. caf.com/bitstream/handle/ 123456789/1091/RED2017-Eng8ene.pdf? sequence $=12 \&$ isAllowed $=$ y\%0Ahttp://dx.doi.org/10.1016/j. regsciurbeco.2008.06.005\%0Ahttps: //www.researchgate.net/ publication/305320484_SISTEM_ PEMBETUNGAN_TERPUSAT_ STRATEGI_MELESTARI

(7) Ministerio de Salud Lima-Perú Dirección General de Salud de las Personas. Impacto socioeconómico de la tuberculosis en el Perú 2010. [Consultado el 11 Nov 2020]. Disponible en: http://bvs.minsa.gob.pe/ local/minsa/1820.pdf

(8) Colombia - Ministerio de Salud y Protección Social. Plan estratégico hacia el fin de la tuberculosis, 2016-2025 (Internet). 2017 [Consultado el 28 Nov 2020]. Disponible en: https://www.minsalud.gov.co/ sites/rid/Lists/BibliotecaDigital/ RIDE/INEC/INTOR/Planestrategico-fin-tuberculosiscolombia-2016-2025.pdf.

(9) OMS - Organización Mundial de la Salud. Objetivos de Desarrollo del Milenio (ODM) [Internet]. 2018 [Consultado 13 Dic 2020]. Disponible en: https: //www.who.int/es/news-room/ fact-sheets/detail/millenniumdevelopment-goals-(mdgs)

(10) OMS - Organización Mundial de la Salud. La estrategia fin de la tuberculosis [Internet]. 2015 [Con- 
sultado el 11 Jul 2019]. Disponible en: https://www.who.int/tb/ Spanish_EndTBStrategy.pdf

(11) Ministerio de la Protección Social. Plan Estratégico Colombia libre de Tuberculosis 2010 - 2015 Para la Expansión y Fortalecimiento de la Estrategia Alto a la TB [Internet]. 2009. [Consultado el 18 Sep 2019]. Disponible en: http://www.ncbi.nlm.nih. gov/pubmed/15003161\%5Cnhttp: //cid.oxfordjournals.org/lookup/ doi/10.1093/cid/cir991\%5Cnhttp: //www.scielo.cl/pdf/udecada/ v15n26/art06.pdf\%5Cnhttp: //www.scopus.com/inward/record. url?eid $=2$-s2.0-84861150233\& partnerID $=$ tZOtx $3 y 1$

(12) Ferreira S, Oliveira HB, Marin L, Ferreira S, Oliveira HB, Marin L. Conhecimento, atitudes e práticas sobre tuberculose em prisões e no serviço público de saúde. Rev Bras Epidemiol. 2013; 16(1):100-113.

(13) Santos MN, Sá AMM. The carrierbeing of tuberculosis in prisons: a nursing study. Esc Anna Nery - Rev Enferm. 2014; 18(2): 350-355.

(14) Adane K, Spigt M, Johanna L, Noortje D, Abera SF, Dinant G-J. Tuberculosis knowledge, attitudes, and practices among northern Ethiopian prisoners: Implications for TB control efforts. PLoS One. 2017; 12(3): e0174692.

(15) Waisbord S. Participatory communication for tuberculosis control in prisons in Bolivia, Ecuador, and Paraguay. Rev Panam Salud Pública. 2010; 27(3): 168-174.

(16) Cruz OA, Flórez EL, Muñoz AI. Conocimientos sobre tuberculosis en trabajadores de la salud en una localidad de Bogotá D. C. Av. Enferm. 2011; XXIX (1): 143-151.

(17) WHO - World Health Organization. Advocacy, communication and social mobilization for TB control: a guide to developing knowledge, attitude and practice surveys. 2008 . [Consultado el 18 Sep 2019]. Disponible en: https://apps.who.int/iris/ bitstream/handle/10665/43790/ 9789241596176_eng.pdf?sequence= $1 \&$ isAllowed $=\mathrm{y}$

(18) Ahumada K, Strba L, Alarcón J, Figueroa J, Niño Y, Pacheco R. Adaptación y validación de un instrumento para medir conocimientos, percepción de riesgo y estigmas sobre tuberculosis. Revista de investigación UNW, 2021; 10(1): 105-129.

(19) Gebrehiwot TT, Tesfamichael FA. Knowledge, Risk Perception and Practice Regarding Tuberculosis Transmission among Long Distance Bus Drivers in Addis Ababa, Ethiopia: A Cross Sectional Study. Ethiop J Health Sci. 2017; 27(6): 601612 .

(20) Cramm JM, Finkenflügel HJ, Møller V, Nieboer AP. TB treatment initiation and adherence in a South African community influenced more by perceptions than by 
knowledge of tuberculosis. BMC Public Health. 2010; 10(1): 72.

(21) Figueroa JE, Hoyos DM, Rodas A, Luna L, Salamanca L, Valencia S, et al. Conocimientos sobre la tuberculosis en usuarios del transporte masivo en Santiago de Cali, Colombia. Interdiscip J Epidemiol Public Heal. 2019; 1(2): 1-7.

(22) Nyasulu P, Sikwese S, Chirwa T, Makanjee C, Mmanga M, Babalola JO, et al. Knowledge, beliefs, and perceptions of tuberculosis among community members in Ntcheu district, Malawi. J Multidiscip Healthc. 2018; 11: 375-389.

(23) Arroyo L, Sánchez L, Bonne M, Pérez H, Armas L. Conocimiento y percepción sobre tuberculosis en el municipio Habana Vieja. Rev Cubana Med Trop. 2012; 64(3): 268-278.

(24) Arroyo JS, Pinzón LF, James J, Gómez DA, Cendales A. Afrocolombianos, discriminación y segregación espacial de la calidad del empleo para Cali. Cuadernos de Economía, 2016; XXXV(69): 753-783.

(25) Bruyneel S, Urréa F, Barbary O, Ramirez HF. Afrocolombianos en el área metropolitana de Cali: estudios sociodemográficos. CIDSE, Centro de Investigaciones y Documentación Socioeconómica, 1999; (38): 1-96.

(26) Muegues SI, Núñez C, Luna L, Pacheco R. Prevalencia y determinantes de tuberculosis pulmonar en un centro carcelario de Cali Colombia,
2013-2014. Interdiscip J Epidemiol Public Heal. 2018; 1(2): 1-7.

(27) Zarate E, Lobón I, Saavedra C, Castañeda María. Tuberculosis en nuevos escenarios: establecimientos penitenciarios. An. Fac. med. 2005; 66(2): 148-158. 